\title{
LOS JÓVENES ESPAÑOLES ENTRE LA INDIGNACIÓN Y LA DESAFECCIÓN POLÍTICA: UNA INTERPRETACIÓN DESDE LAS IDENTIDADES CIUDADANAS
}

\author{
MARÍA LUZ MORÁN* \\ JORGE BENEDICTO**
}

\begin{abstract}
RESUMEN
Este trabajo analiza la reciente evolución de la implicación política de los jóvenes españoles desde la perspectiva de las identidades ciudadanas, considerando que su desafección y activismo son manifestaciones extremas de un complejo proceso dominado por sentimientos de injusticia e indignación. Empleando una metodología cualitativa, se muestra cómo surgen nuevas vías de expresión y ejercicio de la condición ciudadana en las que lo colectivo se re-define como un encuentro de singularidades, y las emociones se convierten en el motor de la acción.
\end{abstract}

PALABRAS CLAVE: JUVENTUD, IMPLICACIÓN POLÍTICA, CULTURAS POLÍTICAS

* Doctora en Sociología. Catedrática de Sociología. Departamento de Sociología I. Universidad Complutense de Madrid. España.

E-Mail: mlmoran@cps.ucm.es.

** Doctor en Sociología. Catedrático de Sociología. Departamento de Sociología I. Universidad Complutense de Madrid. España.

E-Mail: jbenedicto@poli.uned.es.

Este texto forma parte del trabajo realizado dentro del Proyecto de Investigación «¿Redefiniendo la ciudadanía? El impacto de la crisis socioeconómica en las bases de legitimación del Estado de bienestar», financiado por Ministerio de Economía y Competitividad (Ref. CSO201230773). 


\title{
OS JOVENS ESPANHÓIS ENTRE A INDIGNAÇÃO E A DESAFEIÇÃO POLÍTICA: UMA INTERPRETAÇÃO A PARTIR DAS IDENTIDADES CIDADÃS
}

\begin{abstract}
RESUMO
Este trabalho analisa a recente evolução da participação política dos jovens espanhóis a partir da perspectiva das identidades cidadãs, considerando que sua desafeição e ativismo são manifestações extremas de um processo complexo dominado por sentimentos de injustiça e indignação. Usando uma metodologia qualitativa, é mostrado como surgem novas formas de expressão e de exercício da condição cidadã nas que o coletivo é redefinido como um encontro de singularidades e as emoções se tornam o motor da ação.
\end{abstract}

\author{
PALAVRAS CHAVE: : JUVENTUDE, PARTICIPAÇÃO POLÍTICA, CULTURAS \\ POLÍTICAS
}

\section{SPANISH YOUTH BETWEEN POLITICAL OUTRAGE AND APATHY: AN INTERPRETATION FROM CITIZEN IDENTITIES}

\begin{abstract}
This work provides an analysis on the recent evolution of political participation of Spanish young people from a citizen identity perspective, regarding their apathy and activism as extreme manifestations of a complex process driven by injustice and outrage. Through a qualitative methodology, new ways of expressions and exercise of the citizen condition are shown, expressing a re-definition of the collective as an encounter of singularities with emotions turning into a driving force for action.
\end{abstract}

KEY WORDS: YOUTH, POLITICAL IMPLICATION, POLITICAL CULTURES 


\section{LA SORPRENDENTE EVOLUCIÓN DE LA IMPLICACIÓN POLÍTICA DE LOS JÓVENES ESPAÑOLES}

EN MAYO DE 2011 los medios de comunicación se hicieron eco de las multitudinarias protestas que comenzaron en la Puerta del Sol de Madrid y prosiguieron en muchas ciudades españolas. A partir de ese momento, el Movimiento del 15-M —o de los «indignados»— pasó a convertirse en una referencia ineludible del ciclo de movilizaciones globales iniciado en Túnez y Egipto, que se completó simbólicamente con Occupy Wall Street a finales de ese mismo año. Lo más sorprendente fue que hasta ese momento no había indicios claros que hicieran pensar en que fuera a producirse una movilización de este tipo a pesar de que la crisis económica y financiera llevaba ya varios años golpeando de forma brutal a la población española.

Los jóvenes, mayoritarios en el origen de las manifestaciones que desembocaron en las acampadas, tampoco habían mostrado una implicación política destacable. Por el contrario, en comparación con sus coetáneos europeos estaban aparentemente desinteresados por las cuestiones políticas. Por ejemplo, en 2005 solo el $22 \%$ de los españoles entre 15 y 24 años manifestaba estar interesado en estos temas, un porcentaje muy reducido en comparación con el 54\% de alemanes o el $43 \%$ de italianos de estas edades. Incluso una juventud tan despolitizada como la británica alcanzaba el $30 \%$ de interés. ${ }^{1}$ Algo similar sucedía con otros indicadores de politización como la baja propensión a asociarse o las elevadas tasas de abstención electoral. Pero tampoco puede obviarse que — con la excepción del voto - la protesta era con gran diferencia la forma de participación que más utilizaban y la que consideraban más eficaz para hacer oír su voz, lo que también los diferenciaba de los jóvenes europeos.

Al mismo tiempo, hasta muy poco antes del 15-M, los medios de comunicación habían difundido una imagen bastante negativa de la juventud española. La presentaban como un colectivo con una cultura individualista y hedonista, más preocupado por el disfrute inmediato, la capacidad de consumo y el éxito individual que por los problemas sociales. Los análisis académicos recordaban las tradicionales debilidades de la cultura cívica española, pero también insistían en los efectos negativos de su elevada dependencia familiar, que fomentaba acti-

1 Los datos españoles corresponden al EJ104 del INJUVE y los del resto de países europeos a la investigación EUYOUPART. 
tudes conformistas y de repliegue individual, alejadas de los compromisos cívicos (Gaviria 2005; Gil Calvo 2002). Se interpretaba, además, que su tendencia a la protesta tenía un carácter básicamente reactivo y que solo era habitual entre quienes tenían más tiempo libre: los estudiantes (Caínzos, 2006). Aunque en los últimos años de la década ya se advertía un incremento de los indicadores de su politización, el panorama era el de una juventud mayoritariamente distante del ámbito político, con un grado elevado de apatía -al igual que sus mayores-, pero también de descontento (Galais, 2012). A esta situación contribuyeron los efectos de las masivas movilizaciones de comienzos del 2000 - contra la guerra de Irak y otras protestas - que fueron sistemáticamente ignoradas por las instituciones, lo que generalizó entre los jóvenes la percepción de que el poder desoía las demandas ciudadanas. Por otra parte, primero el clima de polarización y crispación vivido entre 2004 y 2009, y después la virulencia de una crisis económica muy mal gestionada por todos los actores institucionales desincentivaron la implicación política.

En consecuencia, no es de extrañar la sorpresa que provocó el movimiento de los indignados, no solo por su altísimo seguimiento popular sino también por la gran cantidad de novedades que incorporó. ${ }^{2}$ Las «acampadas» se fueron disolviendo a lo largo del mes de junio, pero durante todo el año 2011 persistió un elevado grado de movilización. La actividad se trasladó a barrios y pueblos, formándose múltiples asambleas locales que mantuvieron activo el potencial de protesta y reivindicación contra las políticas de austeridad (Gil Calvo 2013). ${ }^{3}$

2 Sin ánimo de ser exhaustivos, algunas de estas novedades fueron las acampadas como modalidad de protesta, la organización en red de personas y colectivos sin presencia visible de grupos organizados, la ausencia de líderes y la toma de decisiones de tipo asambleario, el protagonismo de las nuevas tecnologías en el desarrollo y difusión de la movilización, el carácter intergeneracional del movimiento a pesar de la mayoritaria presencia de jóvenes, y, finalmente, el predominio de reivindicaciones sobre el funcionamiento del sistema social y político dirigidas a transformar el modo de definir e interpretar la realidad.

3 Este mantenimiento del potencial de protesta se hizo especialmente visible, por ejemplo, con ocasión de la manifestación multitudinaria que tuvo lugar el 15 de octubre de 2011 en repuesta a la convocatoria mundial United for \#Global Change celebrada conjuntamente en más de 90 países. 
La espectacular irrupción del movimiento de los indignados en la escena política española y la posterior dinámica de movilización despertaron entre algunos analistas un exagerado optimismo sobre la transformación de las bases de los vínculos ciudadanos con la política. El tradicional cinismo político habría dejado paso a una nueva cultura política participativa. En cuanto a los jóvenes —especialmente los de clase media-, sobrecalificados y con problemas de integración que protagonizaron el 15-M-, habrían pasado de considerarse una generación parásita y «pasota» a una «indignada», pero orgullosa de sí misma (Gil Calvo 2013).

La evolución seguida en los cuatro años transcurridos desde las acampadas en las plazas es muy compleja y excede los objetivos de nuestro trabajo. Aunque el sentimiento de indignación que disparó las movilizaciones de 2011 está indudablemente en el origen de algunas transformaciones muy importantes del panorama político español, ${ }^{4}$ la mayoría de los jóvenes sigue moviéndose entre la habitual desafección hacia la política institucional - hoy reforzada por un intenso sentimiento de desconfianza y descontento con el funcionamiento del sistema sociopolítico- - y un renacido activismo que se concreta en distintas acciones de protesta, diferentes tipos de movilización física y virtual e incluso la puesta en marcha de algunos proyectos novedosos de participación en la política institucional. Si queremos entender esta oscilación entre posiciones aparentemente contrapuestas, hay que superar los planteamientos habituales en la investigación sobre la participación política juvenil y llevar a cabo un análisis en profundidad de sus procesos de implicación. Ello significa indagar en las significaciones colectivas que atribuyen a la política y en los fundamentos cívicos de su participación, estrechamente conectados con las transformaciones de la relación juventud-política y de los vínculos cívicos, que se están produciendo en las sociedades de la segunda modernidad.

4 Sin duda, el surgimiento de un nuevo partido, «Podemos», en el año 2014 y el ascenso de «Ciudadanos», una organización hasta ahora con muy poco peso electoral y limitada a Cataluña, no pueden explicarse sin recurrir a este sentimiento de indignación y de rechazo a la política institucional en España. En el momento en que escribimos, a pocas semanas de la celebración de elecciones locales y autonómicas (regionales), el tradicional «bipartidismo imperfecto»-PP/PSOE- vigente en España desde comienzos de los 80 parece estar seriamente amenazado. 
En esta línea, nuestro trabajo propone interpretar la reciente evolución de la implicación política juvenil en España desde la perspectiva de las «identidades ciudadanas». Para ello partimos de la idea de Jones y Gaventa para quienes: «la forma en que las personas se comprenden a sí mismas como ciudadanos es muy probable que tenga un impacto significativo sobre sus derechos y obligaciones y sobre si participan o no, de qué forma y por qué» (2002:13). Por medio del análisis de las identidades ciudadanas juveniles, ${ }^{5}$ trataremos de mostrar cómo la desafección y el activismo son manifestaciones extremas de un complejo proceso dominado por sentimientos de injusticia e indignación, en el que confluyen dos factores de singular importancia. Por una parte, la debilidad de la concepción de «ciudadanía» predominante en la cultura política española, en la que el ciudadano carece del protagonismo que sí tienen, en cambio, las instituciones y las élites dirigentes. De ahí las dificultades para construir socialmente la ciudadanía a través de experiencias concretas y de prácticas vinculadas a los espacios de vida cotidiana de los jóvenes. ${ }^{6}$ Por otra parte, los cambios de los mecanismos por los que estos se vinculan con la esfera pública, derivados de unos procesos de individualización que impulsan formas diferentes de agencia individual y de acción colectiva. El resultado más destacable es la aparición de nuevas vías de expresión y ejercicio de la condición ciudadana, en las que lo colectivo se re-define como un encuentro de singularidades y las emociones se convierten en el motor de la acción.

Pero antes de entrar en el análisis de las identidades ciudadanas de los jóvenes españoles, debemos desarrollar con algo más de detalle nuestra concepción de la relación entre ciudadanía e implicación política que está en la base de toda nuestra argumentación. Este es el propósito del siguiente apartado.

5 En nuestro trabajo, además de datos de diferentes estudios sobre la juventud española, utilizaremos material empírico de diferentes investigaciones que hemos llevado a cabo sobre la construcción de la ciudadanía de los jóvenes españoles desde el año 2000 hasta la actualidad. Sus principales resultados pueden consultarse en Morán y Benedicto (2003, 2008), Benedicto y Morán (2007; 2014), Benedicto (2013; 2014), Benedicto et al. (2014).

6 Esta conceptualización remite a la conocida idea de la ciudadanía vivida (lived citizenship) de Hall y Williamson (1999). 


\section{LOS FUNDAMENTOS CÍVICOS DE LA IMPLICACIÓN POLÍTICA JUVENIL}

Al estudiar la implicación política, los enfoques clásicos de la ciencia y la sociología políticas nos han acostumbrado a pensar desde una perspectiva individualista y cuantitativa. Ello plantea múltiples problemas (Benedicto 2005), pero aquí solo nos interesa subrayar las limitaciones de su concepción de las relaciones entre los ciudadanos y el ámbito de lo político. Se privilegia la dimensión de la puesta en acción, por lo que prácticamente se identifican politización y participación y esta última se concibe como una sucesión de actos, definidos previamente como políticos, que realizan las personas de manera más o menos individual, y cuyo significado e importancia están establecidos a priori. Por consiguiente, apenas se suele prestar atención al proceso y dinámica de la implicación cívica, ni tampoco al contexto de significados colectivos en el que esta se inserta.

$\mathrm{Al}$ contrario de lo que se sostiene desde esta perspectiva, la participación solo adquiere su verdadera relevancia sociopolítica en la medida en que reconozcamos que los procesos de implicación son una de las principales formas por la que los ciudadanos hacen realidad sus vínculos con su comunidad de pertenencia. Aceptar esta premisa implica situar en el centro del análisis el debate sobre la ciudadanía en las sociedades contemporáneas (Isin y Turner 2003) y, más concretamente, recordar que para la perspectiva sociopolítica sus dos componentes fundamentales son la «pertenencia» y la «implicación». Solo considerando ambos elementos se puede comprender cómo se articulan las identidades ciudadanas de los actores y el tipo de vínculos que establecen con la esfera pública.

Recordemos muy brevemente que, ante todo, ser ciudadano significa formar parte de una comunidad determinada. En la ciudadanía contemporánea, la pertenencia se concreta en la atribución de una serie de derechos y obligaciones reconocidos por el Estado. Sin embargo, el análisis sociopolítico ha superado ya hace tiempo la perspectiva formal, centrada en el reconocimiento legal de la condición de ciudadano, y considera la forma en que esos derechos y deberes son puestos en práctica así como el impacto de la desigualdad sobre la capacidad de los ciudadanos para ejercerlos. Pero, además, para formar parte de una

7 Hemos realizado un análisis más detenido de estos dos componentes de la ciudadanía en Benedicto y Morán (2007). 
comunidad política el sujeto debe sentirse miembro de ella, lo que implica la existencia de algún tipo de «nosotros» ciudadano por encima de las diferencias entre los grupos, que permita establecer vínculos de identificación con los otros y con la comunidad en su conjunto.

Por lo que se refiere al segundo componente de la ciudadanía, la implicación, más allá de las habituales consideraciones normativas sobre la necesidad de la misma hay que subrayar que las prácticas cívicas son imprescindibles para que el individuo complete y active su estatus de ciudadano. Ser ciudadano y actuar como tal son requisitos imprescindibles de la condición ciudadana, pero su articulación en las sociedades contemporáneas se torna extraordinariamente compleja. Tal y como afirma Lister (2003), la ciudadanía como estatus y la ciudadanía como práctica interactúan dialécticamente a través de la noción de «agencia». La capacidad de agencia de los individuos se convierte así en la clave que permite superar la tradicional división entre ser un ciudadano en el sentido formal y actuar como tal.

La vinculación entre estatus y práctica resulta especialmente descompensada en el caso de los jóvenes. Al menos en las sociedades occidentales, su problema no es tanto la posesión de derechos como las condiciones de sus transiciones a la vida adulta y la ausencia de recursos adecuados para alcanzar su condición ciudadana. Diversos factores estructurales — clase, género, etnia, etcétera-, institucionales y culturales crean un conjunto de oportunidades y riesgos en base al cual deben negociar su condición de ciudadanos y la posibilidad de llegar a convertirse en agentes activos (Furlong y Cartmel 1997; Gifford, Mycock y Murakami 2014). En la mayor parte de los casos, los jóvenes se convierten en ciudadanos a través de un proceso dinámico, de una forma negociada, por medio de una serie de prácticas concretas que desarrollan junto a otros (tanto se trate de acciones colectivas de protesta o de otras más vinculadas a su vida cotidiana). A través de estas experiencias de ciudadanía, los jóvenes hacen realidad su identidad cívica y descubren nuevas formas de pertenecer y de participar. Los «a priori» de las pertenencias formales dejan paso a un proceso dinámico en el que las identidades toman forma en respuesta a las prácticas y experiencias (Smith et al., 2005:440-441). En ellas, tratan de hacerse presentes en la esfera pública y de desempeñar un papel protagonista en los procesos en los que participan. 
«Presencia»y «protagonismo» constituyen, pues, los dos requisitos imprescindibles para que la ciudadanía juvenil no sea una mera noción ideal asociada al modelo normativo del «buen ciudadano», ${ }^{8}$ sino un elemento de transformación de las relaciones intergeneracionales. Pero para estar presentes, los jóvenes deben disponer de los recursos y competencias necesarios para la acción y neutralizar los obstáculos socioestructurales e institucionales que les impiden tener voz y ser escuchados. ${ }^{9}$ En segundo lugar, el protagonismo supone reconocer la capacidad para transformar los procesos sociopolíticos. En este caso, el principal problema suele tener que ver con las dificultades que tienen para ser reconocidos como interlocutores válidos por parte del poder, y sobre todo para incorporarse a los procesos de toma de decisiones (Benedicto y Morán 2003).

Las condiciones para que los jóvenes estén presentes y sean protagonistas en las arenas públicas guardan también una estrecha relación con la configuración actual de la condición juvenil. Hasta hace poco, la implicación en cuestiones colectivas se identificaba con un modelo de activismo centrado en organizaciones especializadas, congruente con una forma de ser joven bastante pautada y previsible. En la actualidad, las experiencias participativas son mucho más individualizadas, de acuerdo con unas biografías en las que predomina la incertidumbre y la búsqueda de la realización personal. Parafraseando a Norris en su famoso «Democratic Phoenix» (2002), estaríamos transitando de las lealtades a las elecciones.

Sin caer en dicotomías simplistas que enfrentan lo viejo a lo nuevo, es incuestionable que asistimos a una profunda transformación del contexto de la implicación juvenil así como de las formas de su puesta en

8 Ello supone rechazar una concepción de ciudadanía, predominante en muchas políticas sociales y educativas, entendida como un conjunto de normas, valores y reglas que definen un modelo de buen ciudadano (Hart 2009) y proporcionan una identidad estable y definida en la que los jóvenes se integran paulatinamente hasta alcanzar el estatus de adulto. Delanty (2003) alerta sobre los peligros de esta «concepción disciplinaria» de la ciudadanía en la que se educa a los jóvenes.

9 Como se ha demostrado reiteradamente en la investigación empírica, en nuestras sociedades democráticas actuales el principal motivo de su desafección y desinterés hacia la política institucional reside en la extendida percepción de que ésta no se preocupa por sus problemas y que los políticos no hacen caso a sus demandas y necesidades (O'Toole et al. 2003; Henn et al. 2007). 
práctica. Con muy pocas excepciones, aumenta su desinterés — cuando no el rechazo - hacia la política formal, las instituciones encargadas de gestionarla y los políticos. Dicha desafección no implica necesariamente un recelo contra la democracia, pero sí se traduce en una menor participación en los procesos políticos institucionalizados (voto, militancia en partidos políticos, etcétera). Aunque se tiende a interpretar como despolitización juvenil (Kimberlee 2002), la abundante investigación existente no confirma la tesis de un descenso del interés de los jóvenes por los asuntos colectivos, sino que apunta a una reconfiguración de las relaciones jóvenes-política, entendidas esta últimas como experiencias vividas, vinculadas a su propio recorrido vital (O'Toole et al., 2003).

Para entender mejor esta reconfiguración, hay que incorporar algunos fenómenos que tienen una importancia decisiva. En primer lugar, asistimos a una redefinición de la relación individuo-institución. El debilitamiento de la capacidad socializadora de las instituciones (Dubet, 2002) y la menor relevancia que tienen los compromisos organizativos en favor de las estrategias individuales, especialmente entre los jóvenes (Rossi 2009), traen como consecuencia que el individuo se convierta en la referencia última de la acción, mientras la vinculación con la comunidad se singulariza perdiendo parte de su dimensión colectiva. En segundo lugar, hay que tener en cuenta el impacto que tienen las nuevas condiciones en las que se desarrollan las transiciones juveniles sobre sus relaciones con la política institucional. Las crecientes incertidumbres y dificultades a las que tienen que hacer frente en sus procesos de integración (precariedad vital, frustración de expectativas, etcétera) (Côté, 2014; Benedicto, 2014) justifican su alejamiento de un tipo de política en la que predominan los obstáculos para su presencia y protagonismo, y que sienten totalmente alejada de las experiencias que dan sentido a sus recorridos vitales (Harris, Wyn y Younes 2010).

Finalmente, en este nuevo contexto la «socialidad» y el «encuentro con los otros» juegan un papel relevante en la implicación política juvenil. A pesar de la creciente y fomentada individualización de la vida juvenil, o precisamente a causa de ella, su presencia en acciones colectivas está marcada por la necesidad de creación de vínculos con otros ciudadanos, preferentemente jóvenes. En la sociedad de los individuos (Martucelli, 2007), estar con otros, encontrarse en las plazas físicas o virtuales para poder hacer cosas juntos, es uno de los elementos característicos de una participación juvenil que puede ser puntual y revocable, pero que siempre moviliza una gran cantidad de emociones, 
y activa y desactiva vínculos que permiten compartir experiencias, ideas y sentimientos (Perugorría y Tejerina 2013; Benski y Lagman, 2013; Castells 2012).

Todos estos factores influyen en el repertorio de formas a través de las que se plasma la implicación política juvenil. Un repertorio caracterizado por la pluralidad y diversificación (Harris, Wyn y Younes, 2010; Smith et al., 2005), que combina formas nuevas e individualizadas con otras viejas e institucionalizadas (Hustinx et al., 2012) en las que en las que se diluyen las barreras entre lo social y lo político, entre lo presencial y lo virtual. El resultado es un modelo de implicación fluida en el que predominan las estructuras informales y las movilizaciones ad hoc con bastantes opciones de entrada y salida («easy entry and easy exit»). Así los jóvenes optan por un estilo de participación más individualizado y menos orientado grupalmente que les permite sentirse conectados con sus iguales sin las exigencias de las pertenencias institucionales o las identificaciones ideológicas (Vinken y Diepstraten, 2010).

Este nuevo modelo de relaciones más efímeras con la política incide directamente en las formas de expresión de la ciudadanía juvenil en las que coexisten experiencias de presencia activa en la esfera pública con otros momentos de alejamiento y desinterés frente a las cuestiones colectivas. Al tiempo, buena parte de los jóvenes habita en varios mundos políticos a la vez, combinando sus interpretaciones, vocabularios y representaciones para así poder navegar en el espacio público de una manera significativa, de acuerdo con sus necesidades y circunstancias vitales (Benedicto, 2013).

Todo este conjunto de transformaciones origina una situación ciertamente paradójica. Por una parte, los intensos procesos de individualización que afectan especialmente a las trayectorias de las nuevas generaciones, les empujan a considerarse como actores que tienen capacidad de intervenir en los procesos sociales y políticos en los que están insertas sus vidas. La individualización está en el origen del renovado protagonismo de unos jóvenes que crean nuevas modalidades de implicación acordes con sus intereses, estilos de vida y formas de pertenencia. Pero por otra parte, estos mismos procesos, asociados a la fragmentación y atomización, impulsan la creación de identidades alejadas de la conciencia y actividades que implica lo colectivo. Paralelamente, las políticas neoliberales tratan de deslegitimar las diferentes formas de acción colectiva de los jóvenes (White 2007). 
En todo caso, este conjunto de transformaciones está provocando una profunda redefinición de los fundamentos cívicos de la implicación política juvenil. La multiplicidad de identidades, la intermitencia de la implicación o las nuevas formas de acción individual, sin apenas presencia de actores colectivos que canalicen o representen los intereses de los participantes, son expresiones de cómo están cambiando los vínculos que los jóvenes mantienen con su comunidad.

La implicación ya no se define como la adhesión a la ideología de un grupo específico (un Nosotros) sino como una acción dirigida por el interés personal (un Yo) en una causa que puede eventualmente llegar a ser colectiva, pero se origina en el nivel personal (Queniart y Jacques 2004:179).

Si hasta el momento hemos considerado que la implicación juvenil en cuestiones colectivas era la expresión de los vínculos con su comunidad, ahora debemos poner el énfasis en la dimensión personal que es la que - en la mayor parte de las ocasiones - está en el origen de su acción, de la definición de sus pertenencias y de la construcción de sus identidades cívicas.

\section{LAS RELACIONES DE LOS JÓVENES ESPAÑOLES CON LO PÚBLICO: TENDENCIAS DE EVOLUCIÓN}

Con este planteamiento analítico de partida que proporciona una nueva perspectiva para entender las siempre complejas relaciones jóvenes-política, vamos a retomar el caso español y su evolución durante los años transcurridos desde inicios de siglo. Aunque resulte algo esquemático, a efectos de claridad expositiva, diferenciaremos entre dos períodos: el del crecimiento económico y el de la crisis. En el primero, que abarca desde finales de los 90 hasta el 2007/8, la positiva evolución de la actividad económica extendió la sensación de que la integración social de los jóvenes atravesaba una etapa muy favorable. Sin embargo, la realidad era muy diferente. En concreto, aunque la incorporación al mercado de trabajo se aceleró, al mismo tiempo aumentó la precariedad laboral y disminuyeron los salarios medios, hasta el punto de que para muchos trabajar ya no suponía una garantía de emancipación. A ello se añadió el vertiginoso aumento del precio de la vivienda durante estos años. Todos estos obstáculos se vieron reforzados por la práctica inexistencia de políticas dirigidas al fomento de su 
autonomía (Jiménez et al., 2008; Du Boys-Reymond y López Blasco, 2004).

Con distintos énfasis, marcados por los condicionantes estructurales, durante estos años se extendió entre amplios sectores de la juventud española una generalizada frustración de expectativas. Sin embargo, en gran parte de los casos reconocer que tenían unos problemas comunes no se tradujo en discursos colectivos y, por tanto, tampoco generó procesos de politización en el sentido clásico del término. Al contrario, la respuesta más frecuente fue asumir que era inevitable adaptarse individualmente a unas circunstancias adversas frente a las cuales cada cual debía valerse de sus propios recursos. Este discurso de adaptación individual, que se complementaba con la aceptación de su falta de protagonismo colectivo, adoptaba diferentes contornos en unos grupos sociales y otros (Morán y Benedicto, 2003), pero en todos ellos subyacía la idea de un «pacto intergeneracional». Este pacto consistía básicamente en aceptar la subordinación a los adultos, reduciendo sus demandas y exigencias de protagonismo, a cambio de asegurar una integración exitosa en el futuro. Así, los jóvenes asumieron la prolongación de una ciudadanía «por delegación», en la que el disfrute de sus derechos - fundamentalmente de los sociales - seguía realizándose a través de la vinculación con sus progenitores. Al mismo tiempo, aunque se reconocían como sujetos de derechos, retrasaban la asunción de sus deberes y obligaciones cívicas. La máxima, repetida con frecuencia, «no nos toca; no nos corresponde todavía» está perfectamente interpretada por esta joven madrileña.

Pero bueno, no creo que a ninguno nos importe que en ciertas cosas que valga la experiencia, que no nos importa que no se nos tenga en cuenta, ¿sabes? (Grupo de discusión mixto estudiantes universitarios, clase media, 20-22 años, Madrid, 2004).

A cambio, las familias se comprometían a dilatar e intensificar el apoyo a sus hijos/as, especialmente la inversión en su formación. El objetivo fundamental era acrecentar sus capacidades para asegurarles el éxito en sus procesos de transición. Como ya hemos mencionado, la situación favorecía la adopción de estrategias de adaptación individuales; pero, sobre todo, «encerraba» a los jóvenes en unos mundos muy reducidos, los de su vida más próxima: la familia, los amigos, el ocio o el barrio de residencia. Y, al tiempo que el trabajo perdía relevancia en sus proyectos vitales, aumentaba su distancia frente a la esfera 
pública. Como mucho, lo público se entendía como sinónimo de lo comunitario; es decir, de lo más cercano a sus vidas cotidianas.

La consecuencia más negativa de esta falta de autonomía juvenil fue la consolidación de una imagen pública de la juventud como un colectivo «irresponsable y problemático» que, además los propios jóvenes asumían como propia, contribuyendo a mantenerla y reforzarla. Estos dos extractos son muy ilustrativos al respecto:

Es una edad muy mala, lo único que queremos es fiestas. Entonces ese es el problema y que no queremos asentar la cabeza y no queremos ver la realidad.

Pues eso, que nos queremos hacer mayores para determinadas cosas, mayores en cuanto a derechos de yo puedo esto, lo otro; y luego obligaciones queremos las menos... Vivimos como en una burbuja (Grupo de discusión mixto estudiantes de secundaria, clase media/baja, 16-18 años, Madrid, 2002).

Yo veo que los jóvenes con tener un coche, con tener un trabajo y una novia o un novio..., e ir de fiesta... que no se implican socialmente en nada... (Entrevista, mujer, 23 años, clase media, Madrid, 2009).

Aunque no pueda decirse que los jóvenes carecieran de competencias cívicas, ${ }^{10}$ presentan considerables dificultades para definir el ámbito de la pertenencia ciudadana. Por una parte, emplean una concepción muy despolitizada de la ciudadanía, en la que el ciudadano es simplemente el habitante de la comunidad, y el buen ciudadano aquel que cumple con las normas de urbanidad. Además, debido a la debilidad simbólica del «nosotros común» ciudadano en España, la dimensión estatal/nacional de la pertenencia está muy difuminada, frente a un mayor peso de la pertenencia local que, en algunos casos, es compatible con el salto a una ciudadanía global, cosmopolita.

Estos obstáculos para sentirse parte de una colectividad afectan también a la propia formación del «nosotros jóvenes» que siguen expresando en términos de carencias — sin trabajo, sin vivienda, sin

10 En todas nuestras investigaciones -incluso cuando hemos trabajado con los jóvenes de menor edad y con aquellos «en situaciones de riesgo»sorprende que posean un nivel razonable de información sobre cuestiones públicas y un vocabulario político relativamente rico. Además, expresan de forma clara su condición de sujetos de derechos y tienen notables competencias para orientarse en el mundo de la administración pública con el fin de acceder a los servicios y bienes públicos que les afectan directamente (becas, prestaciones sanitarias, ayudas sociales...). 
familia propia - y, en definitiva, como un problema (Morán y Benedicto, 2003).

En el terreno de las experiencias participativas, ${ }^{11}$ el denominador común son las grandes dosis de desafección política que incorporan que, a nuestro juicio, son consecuencia directa del escaso protagonismo colectivo ya señalado. Lo más evidente y recurrente en sus discursos es el desinterés por la política - siempre entendida como institucional-, que con frecuencia se expresa de forma provocadora, incluso exhibicionista.

Yo creo que tiene que cambiar la política sobre todo. A mí la política me parece lamentable, la política de nuestro país, vamos... Yo creo que centran en cosas demasiado sensacionalistas y que a la sociedad no le aportan nada en vez de preocuparse de cosas importantes (Grupo de discusión mixto, jóvenes con transiciones precarias convencionales, 25-29 años, Sevilla, 2010).

El desinterés se mezcla con la desconfianza que suscita la vida democrática institucional, a la que se acusa no tanto de corrupción -un fenómeno con mucho mayor peso años después-, sino sobre todo de estar alejada de sus intereses y preocupaciones, de no prestar atención a sus problemas. A partir de estos dos rasgos, se comprenden las limitaciones que encuentran los jóvenes para politizar sus problemas comunes.

Su escaso protagonismo en la esfera política convencional - no pueden y no quieren formar parte de ella - no excluye su alta valoración de estar y participar en los espacios más próximos a sus vidas cotidianas. El «close to home» se convierte, así, en el ámbito por excelencia en donde su presencia puede tener consecuencias reales, puede «marcar la diferencia». Ello es debido porque es allí en donde comprueban los efectos de su participación a corto plazo, y también porque provoca una satisfacción personal inmediata.

11 De hecho, los jóvenes españoles poseen desde muy pronto experiencias de participación en acciones colectivas, sobre todo en manifestaciones. De acuerdo con los datos de la Encuesta Social Europea, recogidos en el Informe de la Juventud en España de 2012, en el año 2010 el 20,4\% de los jóvenes españoles de 15 a 29 años afirmaba haber participado en una manifestación autorizada, frente al 9,2\% de los jóvenes del resto de los países miembros de la UE. (INJUVE 2012:218). 
Nos encontramos, pues, con que el crecimiento económico no logró resolver buena parte de las dificultades de los jóvenes para lograr su autonomía y, además, fue creciendo la percepción de que la vida democrática estaba afectada por graves problemas. Se percibían claramente las contradicciones que aquejaban a los jóvenes, pero la situación de bonanza económica creó una especie de paréntesis roto bruscamente con el estallido de la crisis (Benedicto y Morán, 2013) que, como era de esperar, afectó al modo en que los jóvenes españoles perciben y expresan su condición de ciudadanos. Aunque persisten parte de los rasgos de la etapa previa, en esta nueva etapa que se inicia en 2008/9 y aún hoy persiste se han producido transformaciones significativas que debemos incorporar al análisis.

El principal cambio es el brusco paso de la promesa diferida de éxito a la «promesa incumplida» que va a caracterizar esta nueva etapa, todo ello dentro de un contexto de precariedad vital que se expresa como bloqueo de expectativas. Esta joven adulta resume perfectamente la sensación de engaño, de incumplimiento de dichas promesas:

Para encontrar trabajo, es la idea que nos vendieron cuando éramos pequeños: tú trabajas, o sea, tú estudia, hijo, haz una carrera, licénciate, y ya si tienes eso te va a venir todo, te va a venir la casa, la novia, el piso, el perro y el coche. ¡No es así! Esa es la idea que nos vendieron... Y ahora todos tenemos no sé cuántas carreras, no sé cuántos máster, idiomas... Y no viene ni la casa, ni el coche, ni el perro ni nada (Entrevista, mujer, 30 años, doctora en sociología en paro, Madrid, 2013).

Las enunciaciones están impregnadas de desconcierto y frustración, pero en algunos casos también traslucen la irritación frente a lo que muchos entienden como una estafa generacional. Ante el bloqueo de su integración social y cívica, siguen prevaleciendo los intentos de adaptación individual mediante estrategias acordes con unos recursos que reconocen ser muy diferentes. Aunque la admisión de la desigualdad de sus transiciones ya estaba presente antes, la crisis la hace mucho más evidente. Por ello, si bien se admite la responsabilidad colectiva en su gestación - se repite una y otra vez «los españoles hemos vivido por encima de nuestras posibilidades»-, se matizan los discursos de responsabilidad individual ante las actuales dificultades y fracasos.

Es que tampoco creo que haya solo un culpable, ¿no?, porque igual en la situación que estamos ahora yo veo que ha sido culpa general, ¿no?, 
porque la gente se ha puesto a comprarse casas... (Entrevista, mujer, 23 años, clase media, Madrid, 2009).

... De todas formas, el modelo de vida que se nos impone ya no es asequible... Consumir, usar y tirar... (Relato autobiográfico, mujer, 26 años, estudiante de Máster de Sociología, Madrid, 2013).

Se extiende, así, una acentuación de la inevitable pérdida de protagonismo en la vida pública, si es que alguna vez la tuvieron. Su marginación genera una frustración que, en ciertos momentos, se torna en indignación. Al tiempo, son mucho más conscientes de los riesgos a los que se enfrentan, en la medida en que el pacto generacional se ha desvanecido.

Los jóvenes emplean diversas estrategias para hacer frente a esta nueva situación, que definen como muy dura. Sus discursos revelan que son plenamente conscientes de cómo en poco tiempo se han visto forzados a modificar, adaptar o a renunciar a sus proyectos de futuro, a sus expectativas (Benedicto et al., 2014). Así, en un panorama que, a primera vista, parecería definirse simplemente por la acentuación de su tradicional desafección frente a lo público, surgen algunos rasgos inéditos de sus identidades ciudadanas. De una desafección, entendida como alejamiento y desinterés hacia un ámbito de la vida social ajeno a sus preocupaciones, se pasa a otra desafección, entendida como descontento, desconfianza y malestar democrático frente a un sistema social y político que no les ofrece soluciones a sus problemas. ${ }^{12}$

Por ello resulta muy significativo constatar el aumento del interés declarado por los temas políticos (similar en el conjunto de la población), de la exposición a los medios de comunicación convencionales y no convencionales, y la mayor frecuencia de conversaciones políticas con la familia, los amigos y en el lugar de trabajo. ${ }^{13}$ A tenor de estos datos, parece confirmarse la tesis de que el contexto de crisis provoca nuevos procesos de politización, aunque la política institucional sigue provocando sentimientos adversos — antes desinterés y aho-

12 Precisamente este descontento con el funcionamiento del sistema junto al sentimiento de injusticia por las consecuencias de la crisis y la gestión de la misma fundamentan el movimiento de los indignados (Laraña y Díez 2012).

13 De acuerdo con los datos de los Informes de Juventud, entre 2004 y 2012 el grado de interés político juvenil aumentó un 75\%, pasando del 23,2\% al 40,7\% (INJUVE 2012:208-214). 
ra desconfianza- ${ }^{14}$ que se concretan en los partidos, los políticos y las principales instituciones democráticas, ${ }^{15}$ pero también en los sindicatos y otros actores «no convencionales», como las asociaciones o las ONGs. Tras el «boom» del asociacionismo que se produjo en España a finales de la década de los 90 (Ariño, 2007; Morales, 2005), en esta nueva etapa se está asistiendo a una disminución que afecta, sobre todo, a las asociaciones de carácter sociopolítico, y que es especialmente acusada entre la población más joven (Jiménez, 2011; INJUVE 2012).

La acentuación de la impotencia cívica entre los jóvenes, provocada por el devenir de la crisis, no excluye el surgimiento de ciertas formas de politización, aunque sí condicione su desarrollo y sus concepciones y prácticas de ciudadanía. Aun así, incluso cuando es evidente que predomina la desafección, no se suele producir una salida total —en el sentido de Hirschman (1981) — ya que la mayoría de ellos continúa situándose y hablando desde el interior de la comunidad política, aunque en una posición todavía más periférica que en la etapa anterior. (Benedicto et al., 2014)

\section{EL EJERCICIO DE LA CIUDADANÍA JUVENIL: ALGUNAS TENDENCIAS DE CAMBIO}

Este proceso de reconfiguración de las identidades cívicas de los jóvenes que acabamos de analizar no se acaba de entender en toda su profundidad sino atendemos a las nuevas formas en que esas concepciones y compromisos ciudadanos se ponen en práctica y se expresan en la arena pública. Como sostiene nuestra hipótesis de trabajo, la conjunción de unas identidades ciudadanas débiles y una reformulación de los vínculos cívicos con lo colectivo está dando lugar a nuevas formas de ejercicio de la ciudadanía juvenil, compatibles con su presencia en la esfera pública pero marcadas por una ruptura de la vieja lógica de la representación.

14 En un estudio realizado por el CIS en 2011 a jóvenes entre 15 y 29 años, el 40,6\% afirmaba que la política le producía en primer lugar desconfianza, el $16 \%$ aburrimiento y el $15,15 \%$ aburrimiento. Frente a ello, solo el $11,8 \%$ decía que le provocaba interés.

15 De acuerdo con la Encuesta Social Europea, el nivel de desconfianza es muy superior en España que en otros países también muy golpeados por la crisis económica como, por ejemplo, Irlanda y Portugal. 
Ciertamente, no se trata de cambios de última hora, simples consecuencias directas y pasajeras del impacto de la crisis, aunque se hayan exacerbado en los últimos años. Por el contrario, el sentimiento de marginación y las barreras que encuentran para hacerse oír y ser escuchados por los adultos constituyen auténticas experiencias compartidas que vienen repitiéndose desde hace años. Esta sensación de frustración colectiva, perfectamente resumida en el verbatim de estos estudiantes universitarios, determina en gran medida cualquier manifestación de integración cívica por parte de los jóvenes españoles.

Es que al final no te hacen ni caso, no te hacen caso; porque no te consideran como lo suficientemente importantes, ¿sabes?... (Grupo de discusión mixto estudiantes universitarios clase media, 20-22 años, Madrid, 2004).

En este marco, las expresiones cívicas más recientes por parte de los jóvenes ofrecen algunos rasgos singulares. En primer lugar, lo más relevante a nuestro juicio es la creciente relevancia que las emociones tienen en las narraciones que hacen los jóvenes sobre su experiencia pública. Los «discursos de las emociones» (Jasper, 2011) impregnan la forma en que se refieren a sus vivencias ciudadanas, especialmente cuando aluden a su implicación en la política de la protesta. En este caso, es muy habitual que se mezcle la frustración ante las dificultades para «estar presentes» en la esfera pública con la indignación ante la actuación de las elites políticas y el desencanto ante el funcionamiento de las instituciones.

O sea, que tengan miedo de nosotros, me parece que... Lo que pasa es que claro, cuando sales a la calle, sacan las pistolas de goma y te tira. En fin, el recurso de la pataleta... Pero me parece una situación que no va a durar. O sea, dices son políticos, o sea, los políticos que hay ahora mismo son políticos con fecha de caducidad, ¿vale? Me parece que la política está... A mí me encanta la política; o sea, me encanta la política. O sea, soy una persona muy política. Pero también es verdad que estoy muy decepcionado (Entrevista, hombre 28 años, doctorando de clase media, Madrid, 2013).

Otro rasgo a destacar es que la individualización de sus estrategias personales para afrontar los nuevos retos y dificultades que les plantea el entorno se traslada al ámbito público. Y esto es así cuanto menos en dos sentidos diferentes. Por una parte, el ejercicio de los derechos y 
deberes ciudadanos se plantea siempre como una cuestión personal, individual. Da lo mismo que se trate del voto o de los derechos sociales, la referencia colectiva pierde peso en contraste con los argumentos individuales, con los merecimientos de cada uno. Pero también la implicación y el activismo se entienden como actividades más bien personales. De ahí, la insistencia en la satisfacción personal que les producen estas acciones y también la sobrevaloración de «lo cercano», como ámbito que garantiza una pronta verificación de los resultados de su participación y, eventualmente, una rápida recompensa personal. Este giro hacia el ámbito personal permea la forma que adoptan sus discursos. Por eso, no sorprende que sus relatos se enuncien casi siempre en primera persona del singular y que recurran con mucha frecuencia al relato —el storytelling (Polletta 2008) — puesto que es el género más apto para reforzar la centralidad de la experiencia que se erige, así, como criterio clave para la atribución de significados (Dubet, 2010).

Esta centralidad del sujeto y de lo vivido, unida a las ya mencionadas debilidades de algunas dimensiones de las identidades ciudadanas, explica también la frecuencia de los argumentos, relatos y justificaciones en clave ética cuando se plantean su presencia en el espacio público. En buena medida, el vocabulario colectivo de lo político se ve reemplazado por un lenguaje de vidas y experiencias personales, de cariz «psicologizante», en el que las constelaciones de emociones que se ponen en juego (Benski y Langman, 2013) no ocultan las abundantes contradicciones en las que los jóvenes incurren al describir, negociar o cuestionar su vinculación con la esfera política. Al igual que ocurría con las estrategias personales que ponen en marcha en sus vidas cotidianas, en sus expresiones de ciudadanía predomina la flexibilidad de los marcos interpretativos: una mezcla de aceptación de la realidad existente y de rechazo al mal funcionamiento del sistema

El cambio de la posición desde la que se habla, y por lo tanto de cómo se habla, resulta aún más significativo cuando se trata de la participación en movilizaciones colectivas. En estos casos, resulta evidente que el origen de la acción se sitúa en el nivel personal y que el motor que la impulsa, más allá de la referencia a una causa determinada, es la necesidad de establecer redes de contacto con otros, de hacer posible la experiencia puntual de una existencia en común. En esta línea, hay que subrayar - según han puesto de manifiesto varias investigaciones - cómo los indignados del 15-M insistían en que estaban participando en un movimiento de «personas», no de ciudadanos, 
en el que se hacían visibles problemas, y no demandas o reivindicaciones (Perugorría y Tejerina, 2013; Tejerina et al., 2013). ${ }^{16}$ El vocabulario de la política contenciosa, característico de los movimientos sociales, en el que los actores colectivos canalizan los intereses de los participantes deja paso a una redefinición de lo colectivo como un encuentro de singularidades

Como era de esperar, estas nuevas formas de expresar la condición de ciudadano también tienen su reflejo en la dimensión de la implicación cívica. Aunque siempre es difícil establecer principios homogéneos, parece evidente que el voto sigue ocupando un lugar destacado. Éste es concebido como un derecho, pero también como una obligación cívica «que a nuestros padres les costó mucho obtener». A pesar de que desconfían de los efectos reales de su participación electoral, hay que cumplir con ella para ser uno más. Este joven activista de un grupo parroquial resume a la perfección el argumento más repetido por los jóvenes españoles al referirse del voto:

Yo pienso que una persona que no va a votar..., a lo mejor soy muy rotundo..., yo creo que no tiene derecho a quejarse. Yo creo que no participas en esta sociedad... (Entrevista, hombre, 29 años, clase obrera, Madrid, 2009).

Junto al voto, se tienden a destacar las formas más individuales de implicación cívica; fundamentalmente, como hemos mencionado ya, aquellas cercanas a sus vidas cotidianas en las que su presencia puede ser significativa. Se trata de una modalidad cercana a la «ciudadanía de los estilos de vida» (Bennett, 1998), que da prioridad a formas y medios que permiten una implicación directa. Pero, sobre todo, hace posible la libertad de «entrada y salida» que facilita la discontinuidad de su activismo. En definitiva, en el caso español se confirma el argumento expuesto en páginas anteriores de que se está constituyendo un nuevo modelo de implicación más fluido, en el que los jóvenes actúan por medio de una pluralidad de formas, espacios y significaciones.

La participación en este tipo de acciones parece tener como principal objetivo ser reconocidos como interlocutores por el poder, aunque sea de forma esporádica y discontinua. En este sentido, debemos

16 A este respecto, es significativo que uno de los primeros manifiestos difundidos al comienzo de la acampada en la Puerta del Sol comenzara diciendo: «Somos personas que hemos venido libre y voluntariamente...». 
reconocer que la presencia de los jóvenes españoles en los últimos veinticinco años en acontecimientos de protesta - movimiento contra la guerra de Irak, movimiento por la vivienda digna, movimiento estudiantil, movimiento ecológico, plataforma anti-desahucios, movimiento anti-taurino, movimiento de los indignados..., constituye una experiencia generacional intensa que los distingue, además, de sus coetáneos europeos.

Por esta razón, conviene detenerse en el particular significado que poseen para ellos estas actividades de protesta; concretamente, las manifestaciones y las distintas formas de ocupación del espacio público. Diversos estudios sobre movilización colectiva en España señalan que uno de los rasgos distintivos de la vida política española es la «normalización» de la protesta (Jiménez, 2011). Las principales causas de este hecho serían los déficits democráticos de un sistema político muy poco sensible a las demandas ciudadanas, así como la generalización del recurso a las manifestaciones y concentraciones de rechazo al terrorismo. ${ }^{17}$ Estos mismos análisis subrayan los efectos socializadores de la generalización de este repertorio.

Sin embargo, con la excepción de la minoría de activistas tradicionales, el modo en el que los jóvenes entienden su participación en este tipo de acontecimientos difiere en parte de la concepción clásica. Quizá lo más relevante sea que el propio formato de las manifestaciones, ocupaciones o acampadas hace posible soslayar la dimensión colectiva clásica de la implicación cívica. Ya hemos mencionado que, en sus discursos, la movilización se presenta como una expresión de raíz individual y, además, acotada en el tiempo y discontinua. Se trata, así, de una implicación que prescinde de mediaciones organizativas entre el participante y el propio acontecimiento. Una relación directa que hacen posible las NTICS, al permitir la conexión constante, en tiempo real, entre el acontecimiento y el potencial participante (Castells, 2012). Consideremos, por ejemplo, cómo hablan los participantes en un grupo de discusión sobre el papel de internet en sus experiencias de implicación cívica:

17 El punto de partida de esta forma de hacer visible el rechazo al terrorismo estuvo marcado por las multitudinarias manifestaciones que se produjeron tras el secuestro y asesinato por ETA del concejal del PP Miguel Ángel Blanco en julio de 1997. Pero desde finales de la década de los 80, Gesto por la Paz comenzó a convocar concentraciones silenciosas en plazas de muchas localidades del País Vasco después de cada atentado. 
Si por lo menos te llega un mensaje de una manifestación, si no quieres ir, por lo menos los reenvías a todo tu directorio, ¿sabes? Por lo menos por si alguien de los que conoces quiere ir, que vaya. Y ellos harán lo mismo, entonces... Si no voy por lo menos colaboro en que salga la información y se siga expandiendo.

...No, sí, yo creo que... que a las dos [manifestaciones] que he ido ha sido por Internet que me han pasado. También hicimos hace poco un apagón, que se convocó por Internet, de cinco minutos... (Grupo de discusión mixto, jóvenes de origen inmigrante latinoamericano con estudios secundarios, 20-27 años, Sevilla, 2010).

Al mismo tiempo, destaca la dimensión simbólica y expresiva de la participación, muy por encima de la utilitaria. En nuestras investigaciones, se repiten expresiones que atribuyen una escasa efectividad a las acciones colectivas, mientras que lo que se valora realmente es el protagonismo que otorgan a quien participa en ellas. Es este rasgo lo que las convierte en momentos clave de sociabilidad y de aprendizaje político.

Porque allí nada más con ir las cámaras y te graban, ya sale en los medios de comunicación. Con los medios de comunicación, que tiene mucha potencia, ya has hecho algo. Ya ir..., y además personalmente ya has hecho algo; ya has participado y ya has hecho algo. ¿Qué se consigue o no? Yo creo que el $90 \%$ de los casos nunca se consigue nada, pero... (Grupo de discusión mixto, jóvenes con transiciones precarias convencionales, 25-29 años, Sevilla, 2010).

Como anteriormente señalábamos, y también constatan Perugorría y Tejerina (2013) en su análisis del movimiento del 15M español, lo importante es «estar juntos», por lo que la concentración en espacios físicos visibles sigue siendo esencial (Castells, 2012). Son, además, movimientos de emociones, en cuyo origen está la ansiedad, el miedo, la humillación, la indignación y la rabia que comparten jóvenes y no jóvenes a la hora de interpretar sus propias condiciones a través de un «marco de injusticia» (Gamson, 1995). Pero, además, la propia movilización, mientras dura, genera la difusión de otro tipo de sentimientos totalmente contrarios: alegría, entusiasmo, orgullo, esperanza... Unos sentimientos que expresan como personas que ponen en común sus problemas.

En las primeras etapas del 15M, emociones reflejas como la indignación y la rabia, pero también el miedo, la ansiedad y la incertidumbre ya es- 
taban «disponibles» entre los españoles, y fueron «gestionadas organizativamente» por los miembros de DRY... Sin embargo, más adelante estas emociones reflejas se entrelazaron espontáneamente con emociones afectivas (por ejemplo, alegría, orgullo, eficacia, «empoderamiento»), conectadas con compromisos afectivos con personas, ideas y lugares (Perugorría y Tejerina, 2013:433).

\section{CONCLUSIONES}

El amplio corpus de material empírico proveniente de entrevistas y reuniones grupales realizadas con jóvenes españoles en estos últimos diez o quince años que hemos manejado a lo largo de este artículo nos ha permitido poner de manifiesto los cambios que se están produciendo en la vinculación de los jóvenes españoles con lo público, y más concretamente en la forma en que se comprenden a sí mismos como miembros de la comunidad, esto es, como ciudadanos. El análisis de los discursos mediante los cuales expresan sus identidades cívicas y entienden sus prácticas participativas revela cómo, en esta última década o década y media, se ha producido una profunda redefinición de los fundamentos cívicos de la implicación política juvenil.

La crisis sociopolítica e institucional que atraviesa España desde finales de la pasada década ha hecho aún más visible algunos de estos cambios al intensificarse el sentimiento de impotencia cívica y frustración colectiva, la desconfianza en las instituciones políticas establecidas y el malestar democrático, predominantes hoy en las débiles identidades cívicas juveniles. Pero el proceso de transformación que hemos tratado de cartografiar tiene raíces más profundas y tiene que ver con la nueva centralidad que la dimensión personal posee en la definición de las pertenencias y en la concepción de las prácticas. En último término, todo ello termina provocando una puesta en cuestión de la vieja lógica de la representación política ya que las identidades cívicas juveniles se sostienen cada vez más sobre nuevas bases más individualizadas, inciertas e inestables.

Desde una perspectiva más amplia, el análisis del caso español permite resaltar las limitaciones de las concepciones convencionales de la participación política juvenil, así como insistir en la necesidad de seguir profundizando en la compleja combinación entre las transformaciones de los contextos de la integración sociopolítica juvenil, la reconfiguración de sus identidades cívicas y la flexibilización e individualización de las formas en las que concretan sus compromisos 
ciudadanos. En definitiva, la investigación sobre jóvenes y política debería trabajar desde una perspectiva en la que la «otra cara de la moneda» de la desafección y la indiferencia que tradicionalmente se les ha atribuido no es el protagonismo político clásico, tal y como ha mantenido el análisis sociopolítico, sino nuevas formas de expresión de la condición ciudadana, más efímeras y puntuales, en las que los fundamentos y valores tradicionales de nuestras democracias - la igualdad, la justicia, la representación, la concepción de los derechos, etcétera- empiezan a ser reformulados y a adquirir nuevos significados que es necesario analizar en detalle.

MADRID (ESPAÑA), MAYO 2014

RECIBIDO: ENERO 2015

ACEPTADO: FEBRERO 2015

\section{REFERENCIAS BIBLIOGRÁFICAS}

ARIÑO, A. (2007): Asociacionismo y voluntariado en España. Valencia: Editorial Tirant lo Blanch.

Benedicto, J. (2005): «Hacia una política participativa». Zona Abierta $\mathrm{N}^{\mathrm{o}} 106 / 107$.

- (2013) «The Political Cultures of Young People: An Uncertain and Unstable Combinatorial Logic». Journal of Youth Studies, Vol.16, №6. (2014) «La integración sociopolítica de los jóvenes en tiempos inciertos». Società Mutamento Politica, №5 (10).

— y M.L. MORÁN (2003): «Los jóvenes ¿ciudadanos en proyecto?», en Benedicto, J Y M.L. MoRÁn (eds.) Aprendiendo a ser ciudadanos. Madrid: INJUVE.

- (2007) «Becoming a Citizen. Analysing the Social Representations of Citizenship among Young People». European Societies No9 (4).

(2013) «De la integración adaptativa al bloqueo en tiempos de crisis. Preocupaciones y demandas de los jóvenes». En M.L. MORÁN (ed.) Actores y demandas en España. Análisis de un inicio de siglo convulso. Madrid: Los Libros de la Catarata, Colección Investigación y Debate. (2014) «¿Otra clase de politización? Representaciones de la vida colectiva y procesos de implicación cívica de los jóvenes en situación de desventaja». Revista Internacional de Sociología.

BENEDICTO, J., et al. (2014): Transitar a la intemperie: jóvenes en busca de integración. Madrid: Ediciones INJUVE. 
BennetT, W.L. (1998): «The UnCivic Culture: Communication, Identity, and the Rise of Lifestyle Politics». Political Science and Politics, Vol.31, N $\mathrm{N}^{\mathrm{4}}$.

BENSKI, T. y L. LANGMAN (2013): «The effects of affects: The place of emotions in the mobilizations of 2011». Current Sociology, Vol.61, No4.

CAÍnzos, M. (2006): «Participación de los jóvenes españoles en manifestaciones. Comparación con los jóvenes europeos y análisis de sus determinantes». Revista de Estudios de Juventud $\mathrm{N}^{\circ} 75$.

Castells, M. (2012): Redes de indignación y esperanza. Madrid: Alianza Editorial.

CiCCHELli (2012): L'esprit cosmopolite. Voyages de formation des jeunes en Europe. Paris: Presses de Sciences Po.

Côté, J. (2014): «Towards a New Political Economy of Youth». Journal of Youth Studies. Vol.17 (4).

DElanty, G. (2003): «Citizenship as a Learning Process: Disciplinary Citizenship versusCultural Citizenship». International Journal of LifeLong Education, Vol.22 (6).

Du BOYS-REYMOND y A. LÓPEZ BLASCO (2004): «Transiciones tipo yo-yo y transiciones fallidas: Hacia las políticas integradas de transición para los jóvenes europeos». Revista de Juventud, $\mathrm{N}^{\circ} 65$.

DuBET, F. (2002): Le déclin de l'institution. Paris: Seuil.

- (2010) Sociología de la experiencia, Madrid: CIS.

Furlong, A. y F. CARTMEL (1997): Young People and Social Change. Buckingham: Open Univ. Press.

GALAIS, C. (2012): «¿Cada vez más apáticos? El desinterés político juvenil en España en perspectiva comparada». Revista Internacional de Sociología No70 (1).

GAMSON, W.A. (1995): «Constructing Social Protest». En H. Jonston y B. Klandermans (eds) Social Movements and Culture. Minneapolis: University of Minnesota Press.

GAVIRIA, S. (2005): «De la juventud hacia la edad adulta en Francia y en España». Revista de Estudios de Juventud, №71.

GIFFORD, C.A., A. MYCOCK y J. MURAKAMI (2014): «Becoming citizens in late modernity: A global-national comparison of young people in Japan and the UK. Citizenship Studies 18 (1).

GIL CAlvo, E. (2002): «Emancipación tardía y estrategia familiar: El caso de los hijos que ni se casan ni se van de casa». Revista Estudios de Juventud $\mathrm{N}^{\mathrm{o}} 58$.

(2013) «Resistencia contra austeridad. La lucha contra el gran ajuste, en la estela del 15M». En S. AguILAR (ed.) Anuari del conflicto social 2012. Barcelona.

Hall, T. y H. Williamson (1999): Citizenship and Community. Leicester: Youth Work Press. 
HARRIS, A., J. WynN y S. Younes (2010): «Beyond Apathetic of Activist Youth: 'Ordinary' Young People and Contemporary Forms of Participation». Young, Vol. 18, $\mathrm{N}^{\circ} 1$.

HART, S. (2009): «The 'problem' with Youth: Young People, Citizenship and the Community». Citizenship Studies Vol. 13 (6).

Henn, M., M. WeInsteIn y S. HodgKInSON (2007): «Social Capital and Political Participation: Understanding the Dynamic of Young People's Political Disengagement in Contemporary Britain». Social Policy and Society, Vol. 6 (4).

HIRSChMAN, A.O. (1981): Exit, Voice and Loyalty: Responses to Decline in Firms, Organizations and States. Cambridge MA: Harvard University Press.

HustinX, L., L. Meis, F. HANDY y R. CNAAN, A. O. (2012): «Monitorial Citizens or Civic Omnivores? Repertoires of Civic Participation among University Students». Youth \& Society, Vol. 44 (1).

INJUVE (2004): Informe de la juventud en España 2004. Madrid: Instituto de la Juventud.

_ (2012) Informe de la juventud en España 2012. Madrid: Instituto de la Juventud.

IsIN, E. y B. TURNER (2003): Handbook of Citizenship Studies. Londres: Sage.

JASPER, J. (2011): «Emotions and Social Movements: Twenty Years of Theory and Research». Annual Review of Sociology, No37.

Jiménez, B., A. Martin, J. NAvarrete, P. Pinta, R. Soler y A. TAPiA (2008): La emancipación tardía. Transiciones juveniles a la vida adulta en España a comienzos del siglo XXI. Madrid: C.I.S. (Col. Opiniones y Actitudes $\left.\mathrm{N}^{\mathrm{o}} 61\right)$.

JIMÉNEZ, M. (2011): La normalización de la protesta. El caso de las manifestaciones en España. Madrid: CIS. (Col. Opiniones y Actitudes $\mathrm{N}^{\circ} 70$ ).

JONES, E. y J. GAVENTA (2002) Concepts of Citizenship: A Review. Brighton: Institute for Development Studies.

KimberleE, R. (2002): «Why Don't British Young People Vote at General Elections?» Journal of Youth Studies, Vol. 5 (1).

LARAÑA, E. y R. DIEZ (2012): «Las raíces del movimiento 15-M. Orden social e indignación moral». Revista Española del Tercer Sector №20.

LISTER, R. (2003): Citizenship: Feminist Perspectives, Palgrave: Basingstoke.

LocKWOOD, D. (1996): «Civic Integration and Class Formation». British Journal of Sociology 47 (3).

MARTUCCELli, D. (2007): Las sociologías del individuo. Santiago: LOM.

MORALES, L. (2005): «iExiste una crisis participativa? La evolución de la participación política y el asociacionismo en España». Revista Española de Ciencia Política, $\mathrm{N}^{\circ} 13$. 
(2014): La consolidación de la 'matriz cultural' de la democracia en España (1982-1996)». En PÉrez Ledesma M. e I. SANZ (eds) Historia de las culturas políticas en España, Vol. IV.

Morán, M. L. (2001): «Une histoire d'incommunicabilité: récits et culture politique en Espagne et au Pays Basque». En D. CEFAI (ed.) Cultures Politiques, Paris: PUF, 2001.

— y J. BENEDICTO (2003): «Visiones de la ciudadanía entre los jóvenes españoles» Revista de Estudios de Juventud, edición especial 25 aniversario Constitución Española.

— (2008): «Los jóvenes como actores sociales y políticos en la sociedad global». Pensamiento Iberoamericano $\mathrm{N}^{\circ} 3$.

NoRrIS, P. (2002): Democratic Phoenix: Reinventing Political Activism. Cambridge: Cambridge University Press.

O’ToOle, T. D. MARSH y S. JONES (2003): «Political Literacy Cuts Both Ways: The Politics of Non-Participation Among Young People». The Political Quarterly.

PERUGORRÍA, I. y B. TEJERINA (2013): «Politics of the Encounter: Cognition, Emotions and Networks in the Spanish 15M». Current Sociology, Vol. $61, \mathrm{~N}^{\circ} 4$.

Polletta, F. (2008): «Story telling in politics». Contexts, Vol. 7, №4.

QUENIART, A. y J. JACQUES (2004): «Political Involvement Among Young Women: A Qualitative Analysis». Citizenship Studies, 8 (2).

Rossi, F. (2009): «Youth Political Participation: Is This the End of Generational Cleavage?». International Sociology 24 (4).

Smith, N. R. Lister, S. Middleton y L. Cox (2005): «Young People as Real Citizens: Towards an Inclusionary Understanding of Citizenship». Journal of Youth Studies, Vol. 8 (4).

Rossi, F. (2009): «Youth Political Participation: Is This the End of Generational Cleavage?» International Sociology 24 (4).

SZERSZYNSKI, B. y J. URRY (2006): «Visuality, Mobility and the Cosmopolitan: Inhabiting the World from afar». British Journal of Sociology 57 (1).

TEJERINA, B. ET AL. (2013): «From Indignation to Occupation: A New Wave of Global Mobilization». Current Sociology, Vol.61, №4, pp.377-392.

VINKEN, H. y I. DiEPSTRATEN (2010): «Buy Nothing Day in Japan: Individualizing life courses and forms of engagement». Young Vol. 18 (1).

WhITE, R. (2007): «Paradoxes of Youth Participation: Political Activism and Youth Disenchantment». En L. J. SAHA, M. PRINT, K. EDWARDS (eds.): Youth and Political Participation. Rotterdam: Sense Publishers. 